\title{
Pediatric Idiopathic Intracranial Hypertension: A not so Rare and Benign Condition
}

\author{
${ }^{1}$ Ashok K Gupta, ${ }^{2}$ Karan Gupta, ${ }^{3}$ Manish Modi, ${ }^{4}$ Amod Gupta
}

\begin{abstract}
Objective: Pediatric idiopathic intracranial hypertension is an underdiagnosed entity with catastrophic presentations. High index of suspicion with early diagnosis and prompt treatment is the key to successful management. Trans-nasal trans-sphenoid Endoscopic Optic Nerve Fenestration is an effective surgical modality for the reversal of vision loss in pediatric idiopathic intracranial hypertension (IIH).
\end{abstract}

Materials and methods: This is a single center observational prospective case series. Five diagnosed pediatric patients of IIH satisfying the modified Dandy criteria and reported to the out-patient services of otolaryngology, Postgraduate Institute of Medical Education and Research (PGIMER), Chandigarh, India in the year 2012 were included in this study. All children underwent thorough clinical examination, complete Neuroophthalmological work-up including Visual acuity (V/A), Visual field charting (V/F), Fundus venogram and Radiological work-up with MRI for special optic nerve sections in sagittal reconstruction. Cerebro-Spinal Fluid pressure (CSF) measured preoperatively for all children. Standard endoscopic optic nerve Sheath Fenestration was performed on all children. visual improvement was assessed by comparing preoperative ophthalmological findings.

Results: Improvement in vision was taken as a positive outcome. Vision improved in all except one child, who had preexisting optic nerve atrophy.

Conclusion: Endoscopic optic nerve fenestration is an effective minimally invasive procedure to revert visual loss in pediatric idiopathic intracranial hypertension.

Keywords: Pediatric, Idiopathic intracranial hypertension, Optic nerve fenestration.

How to cite this article: Gupta AK, Gupta K, Modi M, G upta A. Pediatric Idiopathic Intracranial Hypertension: A not so Rare and Benign Condition. Clin R hinol An Int J 2014;7(1):5-9.

\footnotetext{
${ }^{1}$ P rofessor, ${ }^{2}$ S enior Resident, ${ }^{3}$ Assistant Professor

${ }^{4}$ Professor and Head

1,2Department of Otolaryngology, Head and Neck Surgery Postgraduate Institute of Medical Education and Research Chandigarh, India

${ }^{3}$ Department of Neurology, P ostgraduate Institute of Medical Education and Research, Chandigarh, India

${ }^{4}$ Department of Ophthalmology, Postgraduate Institute of Medical Education and Research, Chandigarh, India

Corresponding Author: Ashok K Gupta, Professor, Department of Otolaryngology, Head and Neck Surgery, Postgraduate Institute of Medical Education and Research, Sector-12 Chandigarh-160012, India, Phone: +91-1722756763, Fax: +91-1722744401, e-mail: drashokpgi@ hotmail.com
}

\section{Source of support: Nil}

Conflict of interest: None

\section{INTRODUCTION}

Idiopathic intracranial hypertension $(\mathrm{IIH}$, also known as pseudotumorcerebri) is a disorder associated with increased intracranial pressure without clinical or radiological evidence of a space-occupying lesion and with normal cerebrospinal fluid (CSF) constituents. ${ }^{1-5}$ The diagnosis is established according to the modified $D$ andy criteria. ${ }^{6}$ It is well described in the adult population, and is usually associated with female gender, obesity and child-bearing age. The effects of $\mathrm{IIH}$ on the visual system may be catastrophic; approximately $25 \%$ of patients may have significant visual impairment and about $10 \%$ cases with bilateral visual loss. ${ }^{7,8}$

The condition is relatively rare among young children and only few studies have described it in the pediatric population. Some studies have suggested that the clinical profile of children with IIH may be different than that of their adult counterparts and that the precipitating factors may be considerably different as well. ${ }^{7,9,10}$ The condition is rather underdiagnosed in the pediatric age group as the usual risk factors of obesity, child-bearing age are usually not found associated. IIH in pediatric age group tends to be aggressive both due to delay in diagnosis as well as due to the disease progression as well. Pediatric $\mathrm{IIH}$ is diagnosed late could have devastating repercussions for the child in terms of vision deterioration.

Treatment modalities vary from nonsurgical to surgical options. N onsurgical options include use of acetazolamide, furosemide, oral corticosteroids, oral glycerol and repeated lumbar punctures. ${ }^{11}$ Surgical modalities vary from minimally invasive endoscopic optic nerve sheath fenestration (ONSF) to shunting procedures to medial or lateral orbitotomy and transconjunctival optic nerve sheath decompression to subtemporal craniotomy. ${ }^{12-17}$

\section{MATERIALS AND METHODS}

This is a prospective single center observational study conducted in the department of otolaryngology, Postgraduate Institute of M edical Education and Research (PGIMER), Chandigarh, India from 2012 to 2013 including 5 cases of 
proven pediatric $\mathrm{IIH}$ fulfilling the modified $\mathrm{D}$ andy's criteria (Table 1).

A fter getting approval from the institutional review board (IRB), children younger than 15 years, normal brain $M R$ imaging with or without signs of elevated intracranial pressure, intracranial pressure greater than $250 \mathrm{~mm} \mathrm{H}_{2} \mathrm{O}$ with normal cerebrospinal fluid content, edema of the optic disk, and a nonfocal neurologic examination were considered.

A part from routine blood investigations all the children were subjected to an initial preoperative complete neuroophthalmological work-up including CSF pressure measurement, visual acuity, fundoscopy, perimetry visual field charting to know the pattern of visual field defect and fundus venogram. Patients were subjected to computed tomography (CT) scan and magnetic resonance imaging (M RI) of the brain to rule out any neurologic factors of raised intracranial pressure (ICP). M RI imaging with special sagittal sections for optic nerve to see the characteristic findings of flattening of posterior orbit dilated tortuous optic nerve. M R venography was performed in all cases to look for any venous sinus abnormality, and CT scan of the paranasal sinuses was performed to look for the D eL ano type of optic nerve and the degree of sphenoid pneumatization.

All children wereassessed for postoperative improvement in vision and perimetry. Postoperative assessment of CSF pressure was performed in all the cases. The cases were followed up for a period ranging from 3 to 18 months to look for further improvement or deterioration in vision or perimetry.

\section{SURGICAL TECHNIQUE}

Standard transnasal transethmoidal endoscopic optic nerve fenestration was performed using 0 degree rigid nasal endoscope under hypotensive general anesthesia. Skeletonization of ethmoidal gallery after medialization of middle turbinate was done to expose the lamina paparacae (LP) followed by removal of posterior $1 / 3$ rd of $L P$ to get optic tubercle into the view. Further optic tubercle was drilled to expose the optic canal and internal carotid artery along with carotico-optic recess in the lateral wall of sphenoid

Table 1: Diagnostic criteria of idiopathic intracranial hypertension (modified Dandy's criteria)

- Symptoms of increased ICP (headaches, nausea, vomiting, transient visual al obscurations or papilledema)

- No localizing findings in neurological examinations (except for false localizing signs such as abducens or facial palsies)

- Awake and alert patient

- Normal CT/MRI findings without evidence of dural sinus thrombosis

- ICP of $250 \mathrm{~mm} \mathrm{H} \mathrm{H}_{2} \mathrm{O}$ with normal cerebrospinal fluid cytological and chemical findings

- No other cause of increased ICP found
(Fig. 1A). Thin bone of optic canal was removed using periosteum elevator in its entire course till it enters into skull base (Fig. 1B). Two separate windows were made one at the level of annulus of Zinn and the second one in the course of optic nerve to incise the sheath without damaging the nerve fibers (Fig. 1C). Out flow of CSF was seen.

\section{Criteria for Improvement}

Reversal of vision loss, relief from headacheand improvement in visual acuity of two or more scales on the Snellen's chart was taken as improvement.

\section{RESULTS}

All children fell between 6 and 14 years with a mean age of 12 years. O ut of fifteen, 4 were girls and 1 boy. Variable degree of headache and diminished vision were the two chief presenting complaints in all children which ranged from blurring of vision to complete loss of vision. Finger counting close to the face was seen in 4 children and bilateral vision loss seen in 1 .

\section{Ophthalmological Findings}

A ll children had diminished vision at the time of presentation. Three had finger counting at one foot, one at close to face; one child had bilateral vision loss. Papilledema was bilateral in four children and one had optic nerve atrophy on fundus examination.

On visual field charting (Perimetry), nasal scotomas were seen in 3 and concentric field constriction seen in 1 child.

\section{Visual Evoked Potentials}

There was diminished amplitude and increased latency in 4 cases with absent wave form in 1 patient.

CSF pressure measurement showed a range of 250 to $300 \mathrm{~cm}$ of water with a mean of $270 \mathrm{~cm}$ of water.

\section{Radiological Findings}

Typical flattening of post part of orbit with dilated tortuous optic nerve (Fig. 2) was seen in 4 cases. Three patients were found to have E mpty sella (Fig. 3).

Four children out of 5 had reversal of vision loss. Only 1 child did not improved because of underlying optic atrophy.

All children were followed-up after 1, 3 months and then 6 monthly once for assessment of vision and fundus venogram, none of them showed any recurrence.

\section{DISCUSSION}

Idiopathic intracranial hypertension is the most recent of a number of names for the clinical syndrome of elevated 

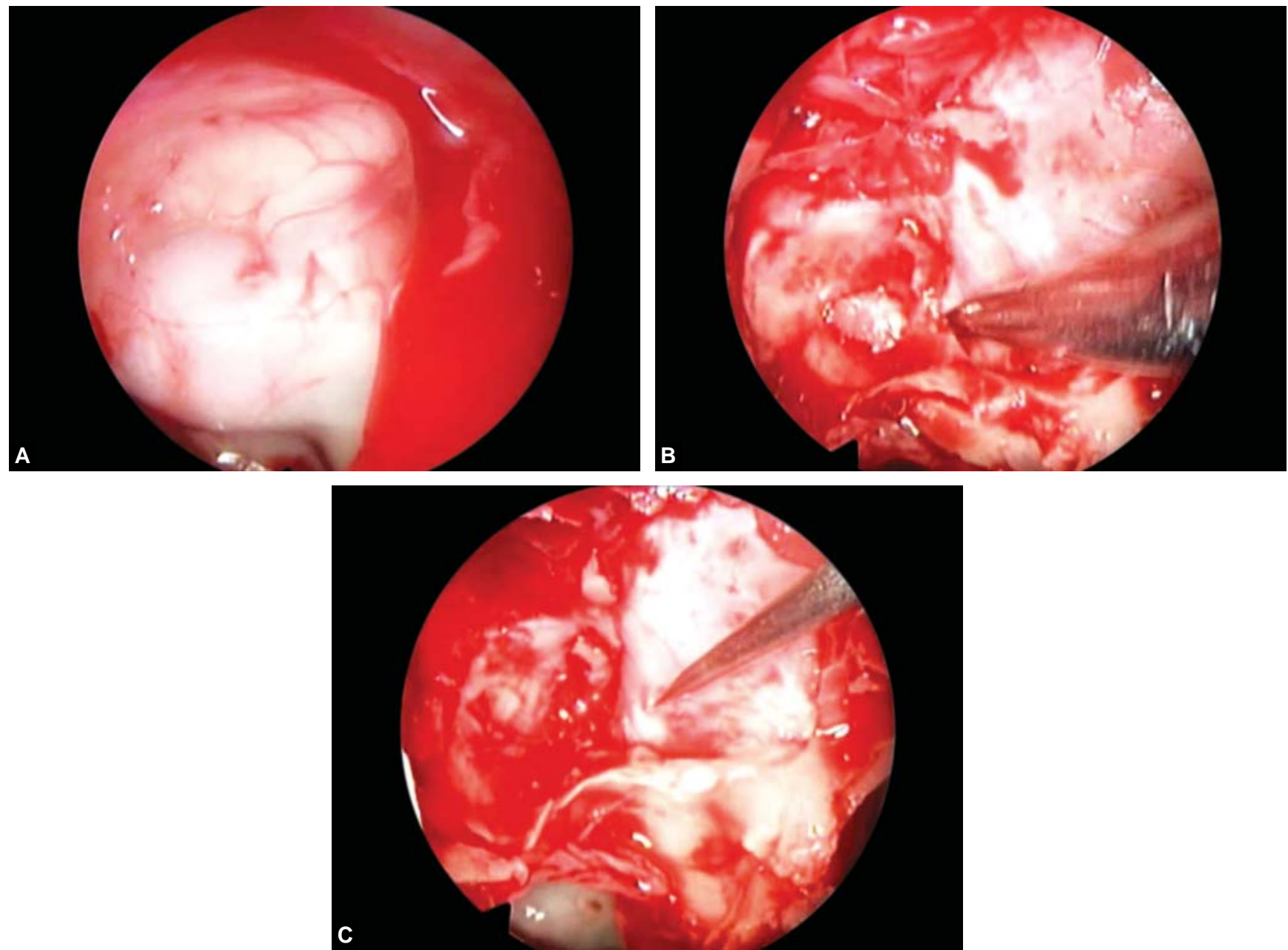

Figs 1 A to $C$ : $(A) 0^{\circ}$ rigid nasal endoscopic view showing widened anterior face of sphenoid to make posterior ethmoids and roof of sphenoid continuous, (B) $0^{\circ}$ nasal endoscopic view showing a blunt freer elevator being used to remove the posterior $1 / 3 \mathrm{rd}$ of Lamina papyracea, $\sim 1$ to $1.5 \mathrm{~cm}$ anterior to the junction of the posterior ethmoids and sphenoid, (C) $0^{\circ}$ nasal endoscopic view of two separate windows using a sharp sickle knife are made $\sim 3 \times 2 \mathrm{~mm}$, one just behind, $\sim 1 \mathrm{~mm}$ behind the level of annulus of Zinn and the second one in the course of optic nerve $\sim 3$ to $4 \mathrm{~mm}$ before the nerve enters the skull base, by incising the sheath without damaging the nerve fibers

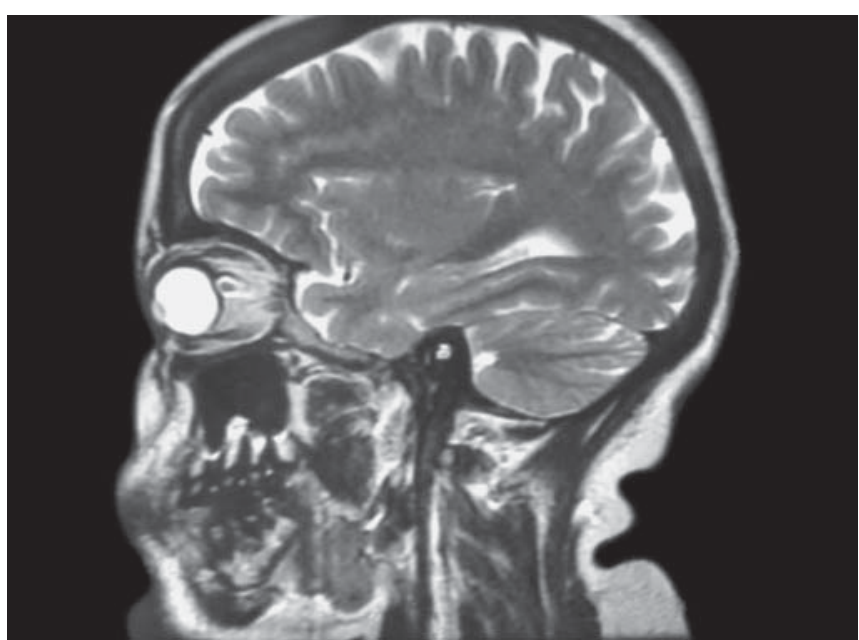

Fig. 2: MRI (T2-weighted) sagittal image showing tortuous and dilated optic nerve with flattening of posterior part of globe

intracranial pressure, without enlargement of the cerebral ventricles and in the absence of space occupying lesions. The German physician Heinrich Quincke (1983) published what is widely regarded as the first description of the condition, calling it 'meningitis serosa'. ${ }^{18}$

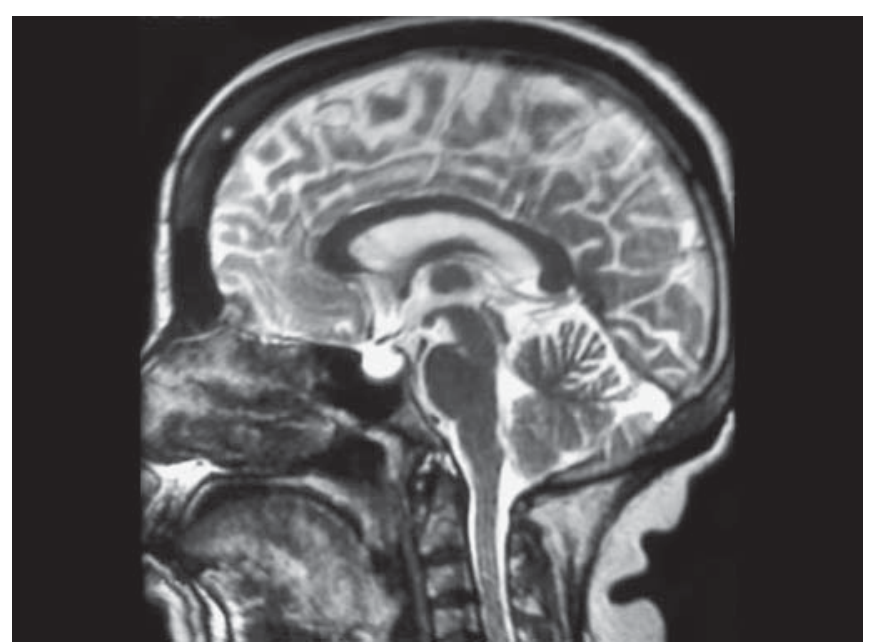

Fig. 3: MRI (T2-weighted) sagittal image showing empty sella

In 1937, the A merican neurosurgeon Walter Dandy (1937) described 22 cases of 'intracranial pressure without brain tumor and is credited with the first diagnostic criteria for the condition. The diagnostic criteria have undergone several modifications over the years. Strict criteria now exist 
to ensure that a diagnosis of $\mathrm{IIH}$ is only applied to patients in whom all other 3 causes of intracranial hypertension have been excluded. ${ }^{6}$ These are shown in Table 1 and are often referred to as 'modified Dandy criteria'.

Idiopathic intracranial hypertension is predominantly a disease of young adult females. The condition does occur in childhood, although no large epidemiological studies in this group of patients have been carried out. It is rare in pre-pubertal children and has different characteristics to the adult form, including no apparent predilection for obese females. ${ }^{19,20}$ A mong older teenage children, however, the rates of obesity seem to mirror those of the adult IIH population. ${ }^{21}$ Y ounger children with IIH appear to represent a unique group, leading to the possibility of different mechanisms underlying the childhood form of the disease. Our study does not show any relationship of obesity with incidence of $\mathrm{IIH}$.

Headache has consistently been shown to be the most common symptom of $\mathrm{IIH}$, occurring in 68 to $98 \%$ of patients and featuring as the presenting complaint in many. It appears to be less common among children with the condition, who may frequently present with other signs such as irritability or visual failure. This is one of the major reasons for the delay in diagnosis of this condition.

Due to lack of the traditional risk factors like association with female gender and obesity and unusual absence of headache in these children there is a substantial delay in diagnosis of $\mathrm{IIH}$ in these patients. This delay in diagnosis and subsequently early treatment is very detrimental for the visual outcome in these patients.

The various treatment modalities used in children have included nonsurgical corticosteroids, acetazolamide, furosemide, repeated lumbar punctures, and surgery including lumboperitoneal puncture and optic nerve sheath fenestration. M ost cases respond to nonsurgical management. The goals of treatment are symptom relief and preservation of vision.

A cetazolamide, a carbonic anhydrase inhibitor, is perhaps the most commonly used drug of first choice in various doses of $0.5 \mathrm{gm} /$ day to $1 \mathrm{gm} /$ day to resolve papilledema and CSF pressure. 22,23

Surgical management is indicated in those with deteriorating visual function and/or severe incapacitating headaches interfering with daily activities despite vigorous medical management. Surgical options vary from neurosurgical approaches to ocular approaches to rhinological approaches.

Currently, lumboperitoneal shunting (LPS) and ONSF are the two surgical procedures employed.

LPS effectively lowers intraventricular pressure and relieves headaches and papilledema. U nfortunately, it is fraught with problems most commonly shunt obstruction and low pressure headaches. Other complications include meningitis, peritonitis, sudden vision loss, acquired cerebellar tonsillar herniation, syringomyelia, lumbar radiculopathy and death. ${ }^{13}$ The mortality and morbidity was 9 to $17 \%$.

Optic nerve sheath fenestration is currently the favored treatment for successful relief of papilledema and rapid reversal of vision loss in most cases of $\mathrm{IIH}$ who deteriorates despite of maximum medical treatment. The mechanism by which optic nerve sheath fenestration helps is by reducing the transmitted pressure on the optic nerve. The CSF pressure is reduced locally over the nerve, and the vision improves and does not deteriorate further.

Patients with $\mathrm{IIH}$ and vision threatening papilledema should be offered ONSF without delay. R esults are favorable in terms of visual outcome, there is an infrequent need for repeated surgery, and in expert hands the procedure is safe with few intraoperative or postoperative complications and no reported mortality.

\section{CONCLUSION}

Idiopathic intracranial hypertension in pediatric age group leads to visual loss. IIH in pediatric age group is an underdiagnosed cause for vision loss. Endonasal endoscopic optic nerve sheath fenestration is a minimally invasive and effective al ternate to prevent vision loss as compared to other surgical modalities like subtemporal craniotomy; Lateral orbitotomy, etc.

\section{REFERENCES}

1. Kesler A, Goldhammer Y, Gadoth N . D o men with pseudotumorcerebri share the same characteristics as women? A retrospective review of 141 cases. J Neuro-ophthalmol $2001 \mathrm{M}$ ar;21(1):15-17.

2. Digre KB, Corbett JJ. Idiopathic intracranial hypertension (pseudotumorcerebri): a reappraisal. N eurologist 2001;7:2-6.

3. Radhakrishnan K, A hlskog JE, Cross SA, et al. Idiopathic intracranial hypertension (Pseudotumorcerebri). Descriptive epidemiology in Rochester, M inn. 1976 to 1990. A rch N eurol 1993 J an;50(1):78-80.

4. A hlskog JE, O'N eill B P. Pseudotumorcerebri. A nn Intern M ed. 1982 A ug;97(2):249-256.

5. Radhakrishnan K, A hlskog JE, Garrity JA, K urland LT. Idiopathic intracranial hypertension. M ayo Clin Proc 1994 Feb; 69(2):169-180.

6. Friedman DI, Jacobson DM . Diagnostic criteria for idiopathic intracranial hypertension. N eurology 2002 N ov 26;59(10):14921495.

7. Lessell S. Pediatric pseudotumorcerebri (idiopathic intracranial hypertension). Surv Ophthalmol 1992 Nov-Dec;37(3):155-166.

8. Wall M, George D. Idiopathic intracranial hypertension. A prospective study of 50 patients. B rain $1991 \mathrm{Feb} ; 114(\mathrm{Pt} \mathrm{IA} \mathrm{):155-180.}$

9. Babikian P, Corbett J, B ell W. Idiopathic intracranial hypertension in children: the lowa experience. J Child N eurol 1994 A pr;9(2):144-149.

10. Baker RS, Baumann RJ, Buncic JR. Idiopathic intracranial hypertension (pseudotumorcerebri) in pediatric patients. Pediatr Neurol 1989 Jan-Feb;5(1):5-11.

11. Corbett JJ, Savino PJ, Thompson HS, et al. Visual loss in pseudotumorcerebri: follow-up of 58 patients from 5 to 41 years 
and a profile of 14 cases with permanent severe visual loss. A rch Neurol 1982 A ug;39(8):461-474

12. Gupta AK, Ganth M G, Gupta A. M odified endoscopic optic nerve decompression in idiopathic intracranial hypertension. J Laryngol O tol 2003 J un;117(6):501-502.

13. Gupta AK, Gupta A, K umar S, et al. Endoscopic endonasal management of pseudotumorcerebri: is it effective? Laryngoscope 2007 Jul;117(7):1138-1142.

14. Shurtleff DB, Stuntz JT, Hayden PW. Experience with 1201 cerebrospinal shunt procedures. Pediatr Neurosci 1985-1986; 12(1):49-57.

15. Corbett J J, N erad JA, Tse DT, A nderson RL. Results of optic nerve sheath fenestration for pseudotumorcerebri. The lateral orbitotomy approach. A rch Ophthal mol 1988 Oct; 106(10):13911397.

16. Galbraith JE, Sullivan JH. Decompression of the perioptic meninges for relief of papilloedema. A m J Ophthalmol 1973 Nov;76(5):687-692.
17. Wilson DH, Gardner WJ. B enign intracranial hypertension with particular reference to its occurrence in fat young women. Can M ed A ssoc J 1966 J ul 16;95(3):102-105.

18. Quincke H. M eningitis Serosa. Sammi K lin Votr 1893;67.

19. Cinciripini GS, D onahueS, B orchert M S. I diopathic intracranial hypertension in prepubertal pediatric patients: characteristics, treatment and outcome. Am J Ophthalmol $1999 \mathrm{Feb} ; 127(2)$ : 178-182.

20. Rose $A$, Matson DD. Benign intracranial hypertension in children. Pediatrics 1967 Feb;39(2):227-237.

21. Rowe F, N oonan C. The prevalence of overweight and obesity in a childhood population of idiopathic intracranial hypertension. Neuro-ophthal mol 2002;28(2):87-93.

22. Symonds C. Otitic hydrocephalus. Neurology 1956 Oct; 6(10): 681-685.

23. Buchheit WA, B urton C, Haag B, et al. Papilloedema and idiopathic intracranial hypertension. N Engl J M ed 1969 A pr 24; 280(17):938-942. 